\title{
HIV clearance in an infant?
}

SIR - Infants born to HIV-infected mothers acquire maternal antibody transplacentally, rendering the interpretation of serological assays difficult. Secure diagnosis of perinatal infection should include investigation both of a maternal sample and of two or more infant samples, using a combination of assays for the virus (culture, genome and antigen detection) and for the evolving serological response (or its absence) in the infant. For absolute security, results must be concordant at any point in the sampling schedule.

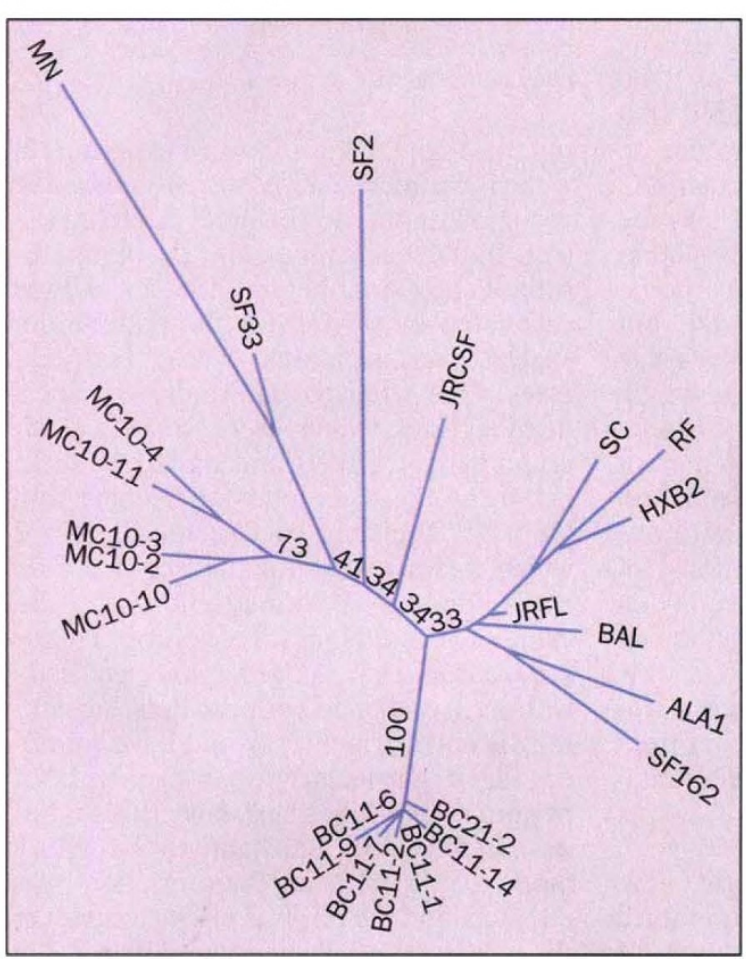

Phylogenetic analysis of mother/child env sequences. DNA sequences corresponding to those published in ref. 1 (BC, infant sequences; MC, maternal sequences; other sequences represent unrelated laboratory strains of HIV) were obtained from GenBank and aligned using CLUSTAL. Modifications to improve the alignment were made by hand. This alignment was used as input for PHYLIP programs. Genetic distance was estimated using the six-parameter model of J. Felsenstein (PHYLIP program DNADIST, version 3.5c; distributed by the author, Department of Genetics, University of Washington, Seattle), and these distances were used to construct a neighbour-joining tree. The robustness of the tree was assessed using the bootstrap resampling method with 100 replications.

Following the media attention surrounding the reported clearance of HIV infection in a perinatally infected infant by Bryson et al. ${ }^{1}$, it is worth noting that on the data presented, the baby in question would not have met the criteria of infection adopted at some hospitals in the UK, although we accept that they do meet the standards set down by others $^{2-4}$.

The diagnosis by Bryson et al. hinges on virus being cultured from peripheral blood mononuclear cells (PBMCs) on two occasions, 19 and 51 days after birth, any other means, and the only positive 33 days was unconfirmed by virus culresults do not constitute a concordant Because contaminated cultures and false-positive PCR results are an occupational hazard, we consider a baby to be infected with HIV only when PBMCs and/or plasma taken from the infant on two separate occasions are both cultureand PCR-positive, or to be positive by one of these tests in the face of an evolving antibody response. These stringent criteria remove the element of diagnostic controversy that inevitably surrounds cases of 'viral clearance'. Although ten sequential samples were taken postnatally from the infant described in the paper by Bryson et al., at no time is any one sample shown to be positive by both PCR and culture simultaneously. Hence, it is not unreasonable, in our opinion, to call the baby's virological status into question.

To eliminate the possibility that their results could be explained by inadvertent laboratory contamination of viral isolates, Bryson et al. sequenced independent clones of the V4-C4 part of the viral envelope from the HIV-cultured samples. Sequence similarity between the mother and baby isolates is presented by the authors as evidence of an evolutionary relationship between them. Although the two isolates from the baby are indeed closely related, there is nothing in these sequences to link them with the mother. Our phylogenetic analysis of the published sequences clearly shows that the baby isolates are no more closely related to those of the mother than to many other laboratory strains of HIV (see figure). This, of course, may be the result of selection of a minor variant following culture of the baby's blood sample before sequencing; an extremely rapid turnover in the viral quasispecies of the mother in the year after delivery and before her viral samples were obtained; or a function of the fact that the short V4-C4 region is not ideal for demonstrating genetic relatedness between mother and baby. We urge Bryson et al. to analyse a sequence for which the evolutionary dynamics are more amenable to the demonstration of HIV transmission, such as V3 (ref. 5) or the p17 region of the gag gene ${ }^{6}$.

In view of the above considerations, we believe it is premature to claim clearance of the virus by this or any other infant.

M. O. Mcclure

P. D. Bieniasz

J. N. Weber

Department of GU Medicine,

Jefferiss Research Trust Laboratories,

R. S. Tedder

Department of Medical Microbiology,

Division of Virology,

University College London Medical School, London W1P 6DB, UK

S. O'Shea

J. E. Banatvala

Department of Virology,

UMDS St Thomas' Hospital Campus,

London SE1 7EH, UK

G. Tudor-Williams

Department of Paediatrics,

St Mary's Hospital Medical School,

London W2 1NY, UK

\section{P. Simmonds}

Department of Medical Microbiology,

University of Edinburgh,

Edinburgh EH8 9AG, UK

E. C. Holmes

Department of Zoology,

University of Oxford,

Oxford OX1 3PS, UK

BRYSON AND CHEN REPLY - We disagree with the interpretations of McClure et al. of our recent paper ${ }^{1}$ for a number of reasons. The criteria set out by these authors for the diagnosis of paediatric HIV infection are not typical of those used for most paediatric diagnoses. Our diagnosis of HIV infection is based on the presence of cultured virus from the infant on two separate occasions, consistent with the guidelines established by the NIH AIDS Clinical Trials Group Virology Committee and the Centers for Disease

1. Bryson, Y. J. et al. New Engl. J. Med. 332, 833-838 (1995).

2. Caldwell, M. C., Oxtoby, M. J., Simonds, R. S. Lindegren, M. L. \& Rogers, M. F. Morbid. Mortal. Wkly Rep. 43, No. RR-12 (1994).

3. Paediatric European Network for Treatment in AIDS Trial entry criteria available from the UK Medical Research Council.

4. Rep. 2nd Meet. WHO Informal Working Group on Prevention of Mother to Child Transmission of HIV : Standardization of Techniques and Criteria for the Diagnosis of HIV Infection in Neonates, Rockville, USA WHO, Geneva, 1995).

5. Ahmad, N., Baroudy, B. M., Baker, R. C. \& Chappey, C. J. Virol. 69, 1001-1012 (1995).

6. Holmes, E. C., Zhang, L. Q., Rogers, A. S. \& LeighBrown, A. J. J. infect. Dis. 167, 1411-1414 (1993).

7. AlDS Clinical Trials Group Virology Committee ACTG Virology Manual for HIV Laboratories (Division of AIDS, NIAID/NIH, Bethesda, Maryland, 1994)

8. McIntosh, K. et al. J. infect. Dis. 170, 996-1000 (1994). 